\title{
Are cyclin-dependent kinases 4/6 inhibitors ready for prime time in estrogen-receptor positive metastatic breast cancer?
}

\author{
Sofia Genta $^{1,2}$, Gloria Mittica ${ }^{1,2}$, Gaia Giannone $^{1,2}$, Eleonora Ghisoni $^{1,2}$, Giorgio Valabrega $^{1,2}$ \\ ${ }^{1}$ Department of Oncology, University of Torino, Torino, Italy; ${ }^{2}$ Candiolo Cancer Institute, IRCCS, Fondazione del Piemonte per l'Oncologia (FPO), \\ Candiolo, Italy \\ Correspondence to: Giorgio Valabrega, MD. Candiolo Cancer Institute, IRCCS, Fondazione del Piemonte per l'Oncologia (FPO), Strada Provinciale \\ $142 \mathrm{Km} 3.95,10060$ Candiolo (TO), Italy. Email: giorgio.valabrega@ircc.it. \\ Provenance: This is an invited Perspective commissioned by Section Editor San-Gang Wu (Department of Radiation Oncology, Xiamen Cancer \\ Center, the First affiliated Hospital of Xiamen University, Xiamen, China). \\ Comment on: Hortobagyi GN, Stemmer SM, Burris HA, et al. Ribociclib as First-Line Therapy for HR-Positive, Advanced Breast Cancer. N Engl \\ J Med 2016;375:1738-48.
}

\begin{abstract}
Endocrine therapy is the mainstay of treatment for most hormone receptor positive $(\mathrm{HR}+)$, human epidermal growth factor receptor 2 (HER2) negative breast cancer (BC). Patients with metastatic disease may experience long lasting clinical benefit (CB) despite the line of endocrine therapy. The major limitation of this treatment is primary and, most frequently, acquired resistance. A better understanding of endocrine resistance has resulted in the development of new targeted agents to be integrated with endocrine therapy. The addition of a cyclin-dependent kinases 4/6 (CDK4/6) inhibitor such as ribociclib (MONALEESA-2 trial) or palbociclib (PALOMA-3 trial) to endocrine treatment improved response rate (RR), and prolonged progression-free survival (PFS), but overall survival (OS) data are not yet available. Combination therapy with CDK4/6 inhibitors allows delaying conventional chemotherapy start but increases toxicities and costs. Identification and validation of biomarkers of response that could avoid unnecessary toxicities to patients are therefore essential. Ongoing and future trials will hopefully elucidate the optimal placement of CDK4/6 inhibitors in the treatment of HR+/HER2-BC.
\end{abstract}

Keywords: CDK4/6 inhibitors; breast cancer (BC); endocrine resistance.

Submitted Dec 23, 2016. Accepted for publication Jan 11, 2017.

doi: $10.21037 /$ tcr.2017.02.20

View this article at: http://dx.doi.org/10.21037/tcr.2017.02.20

Breast cancer (BC) is the most common malignancy in women worldwide and estrogen-receptor (ER)-positivity is its hallmark in most patients. Even if outcome has improved over years, ER positive BCs may be resistant ab initio or become resistant to endocrine therapy later on. Many efforts have been made to improve clinical outcome of advanced ER-positive, human epidermal growth factor receptor 2 (HER2)-negative BC patients through the addition of signaling pathways inhibitors such as cyclindependent kinases 4/6 (CDK4/6) inhibitors (ribociclib, palbociclib, abemaciclib). Upon stimulation with mitogenic signals, CDK4 and CDK6 form a complex with their regulatory subunit, cyclin $\mathrm{D} 1$, which phosphorylates the retinoblastoma protein $(\mathrm{Rb})$, reducing its ability to suppress gene transcription (1). Controlled phosphorylation and deactivation of the $\mathrm{Rb}$ protein by the CDK4/6 complex is essential to progression of the normal cell cycle. In BC cells, uncontrolled CDK4/6 activity may result from alterations in the expression of cyclin-dependent kinases and their regulatory mechanisms. As a consequence, cell cycle stimulation leads to a growth advantage and to endocrine resistance (Figure 1).

In a recent article published on the New England fournal of Medicine, Hortobagyi et al. (2) reported the interim analysis of the MONALEESA-2 [Mammary Oncology Assessment of LEE011's (Ribociclib's) Efficacy and 


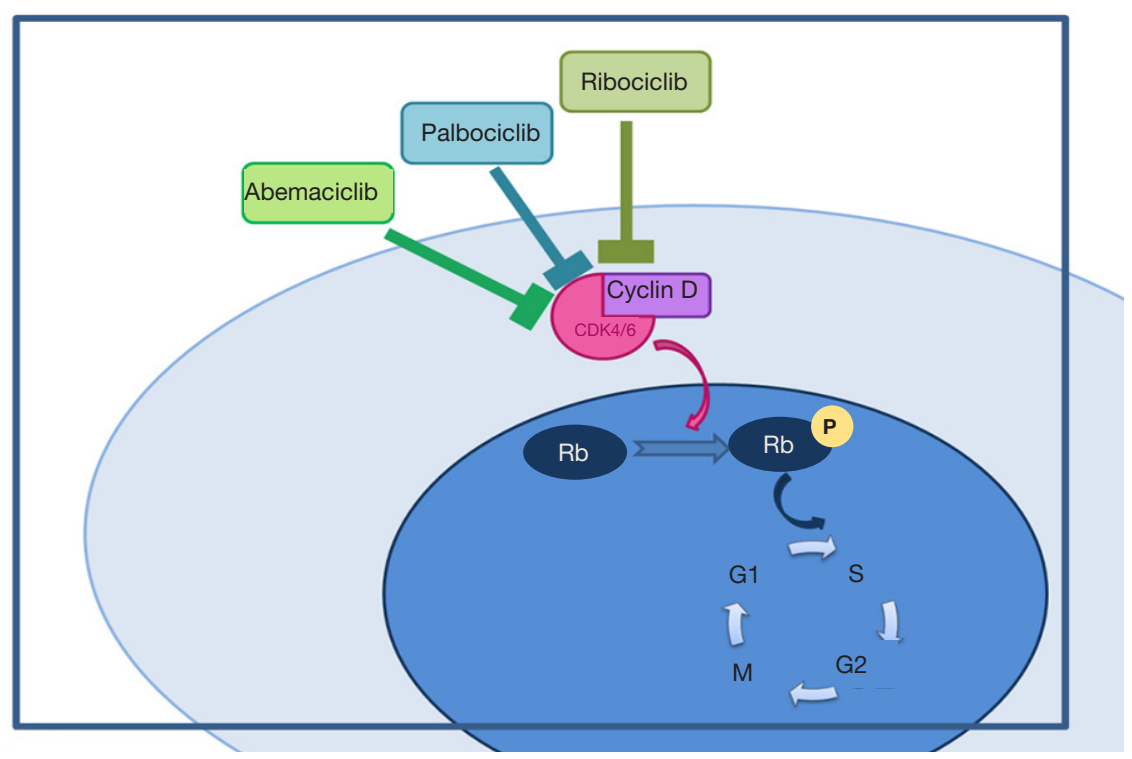

Figure 1 CDK 4/6 inhibitors. CDK 4/6, cyclin-dependent kinases 4/6; G1, cell cycle, G1 phase; G2, cell cycle, G2 phase; M, cell cycle, M phase; $\mathrm{P}$, phosphorilation; Rb, retinoblastoma protein; $\mathrm{S}$, cell cycle, $\mathrm{S}$ phase.

Safety-2] trial. This study included 668 postmenopausal patients treated with non-steroidal aromatase inhibitor letrozole plus the CDK4/6 inhibitor ribociclib or placebo as first-line therapy for metastatic disease. About $30 \%$ of the enrolled patients had newly diagnosed advanced or metastatic disease and near $60 \%$ of the patients had visceral disease. More than $50 \%$ of patients received prior adjuvant endocrine therapy (tamoxifen in about $40 \%$ of patients). Near half of patients had received prior adjuvant chemotherapy. In MONALEESA-2 trial, the addition of ribociclib to letrozole significantly improved progression-free survival (PFS) at 18 months in comparison with letrozole and placebo $(63.0 \%$ vs. $42.2 \%$; hazard ratio for disease progression or death, 0.56 ; $95 \%$ CI, $0.43-0.72)$. Moreover, the vast majority (80\%) of patients treated with the CDK4/6 inhibitor ribociclib obtained a clinical benefit (CB) defined as absence of disease progression. Overall survival has not been reported yet in MONALEESA-2 trial. The most common adverse events were neutropenia $(74.3 \%$ in the ribociclib group and $5.2 \%$ in the placebo group), nausea $(51.5 \%$ and $28.5 \%$, respectively), infections (50.3\% and $42.4 \%$ ), fatigue (36.5\% and $30.0 \%$ ), and diarrhea (35.0\% and $22.1 \%$ ). Nausea, infections, fatigue, and diarrhea were mostly grade 1 or 2 . The most common grade 3 or 4 adverse events were neutropenia $(59.3 \%$ in the ribociclib group and $0.9 \%$ in the placebo group); febrile neutropenia occurred, however, only in five patients $(1.5 \%)$ in the ribociclib group; hypertension $(9.9 \%$ and $10.9 \%)$ and increased alanine amino-transferase level (9.3\% and $1.2 \%)$. QTc interval prolongation was observed only in the ribociclib group (3.3\%). Importantly, about $30 \%$ of the patients enrolled in the MONALEESA-2 trial and assigned to ribociclib required drug reductions. Despite this most patients ( $>90 \%$ ) were able to continue treatment with ribociclib without withdrawal. Overall, serious adverse events (SAE) occurred in 71 patients (21.3\%) in the ribociclib group and in $39(11.8 \%)$ in the placebo group. Of these events, $25(7.5 \%)$ in the ribociclib group and $5(1.5 \%)$ in the placebo group were possibly related to the study regimen. There were 4 deaths $[3(0.9 \%)$ in the ribociclib group and $1(0.3 \%)$ in the placebo group] during treatment. Noteworthy, similar results in terms of safety and efficacy were obtained with the other CDK4/6 inhibitor palbociclib (Ibrance; Pfizer), which was tested in association with fulvestrant the recently published randomized phase III trial PALOMA-3 (3). Moreover, similarly to the MONALEESA-2 trial, in PALOMA-3 trial, dose modifications did not appear to affect PFS. Currently, based on the above study, ribociclib has been submitted for FDA approval in combination with anti-estrogen therapy for advanced HR-positive, HER2 negative BC. Palbociclib received FDA approval last year in the same setting. Several trials with a third CDK4/6 inhibitor (abemaciclib) are ongoing in association with other endocrine therapies such 
as fulvestrant (ClinicalTrials.gov number, NCT02107703) and aromatase inhibitors (NCT02246621) on the basis of an FDA "breakthrough therapy" designation, a process designated to expedite the development and review of drugs that are intended to treat a serious condition and preliminary clinical evidence indicates that the drug may demonstrate substantial improvement over clinically available therapy (4).

Efforts at elucidating the molecular mechanisms of endocrine resistance have revealed a number of targets that act downstream or upstream or crosstalk with ER pathway (4). Consequently, several agents have been developed with the aim of interfering with the ability of ER-positive cancer cells to resist to endocrine therapy such as inhibitors of the PI3K/AKT/mTOR pathway (5) and histone deacetylase inhibitors (6). In particular, the rationale of combining endocrine therapy with therapeutic agents able to prevent, delay or circumvent endocrine resistance has been, and is being tested in several phase II and III trials. In this context, results with CDK4/6 inhibitors from randomized phase III trials MONALEESA-2 and PALOMA 3 could be seen as 'practice changing' on the basis of efficacy. However, beyond the increase in median PFS, the positive studies reported consistent increases in ORR, and a substantial increase in clinical benefit rate (CBR), resulting from more patients achieving long-lasting stable disease with the combined treatment. However, the follow-up of MONALEESA-2 and PALOMA 3 is still too short to adequately evaluate overall survival (OS). This is particularly important because, benefits from adding a biologic agent to endocrine therapy add toxicities and costs. Waiting for OS results, the main question is whether a PFS advantage supports the widespread use of these compounds in the everyday clinical practice and across the whole clinical spectrum of HR+/HER2- MBC patients. Tumor regression and PFS prolongation may have positive effects in patients whose disease is symptomatic, but it may not be true in asymptomatic patients. Furthermore, delaying the use of "conventional" chemotherapy could be seen as a goal of endocrine therapy. However, this prolonged 'chemotherapy-free interval' is filled in with additional toxicities and a clinical management consisting of more frequent hospital visits, lab works, and staging procedures, which are commonly associated with "conventional" chemotherapy. The decision to continue or stop a certain treatment is multifactorial and does take into account not only antitumor activity, but also tumor-related symptoms evolution, treatment-related toxicities, costs, and patient' wishes. With these premises, especially in the era of precision medicine, identification and validation of predictive biomarkers that could allow treatment tailoring and toxicity sparing are really crucial. Nowadays, however, HRs and HER2 are the only biomarkers used to select patients for combinations of biologics with endocrine therapy. Most of the clinical trials that have led to the registration of biologics in association with to endocrine therapy, included extensive biomarker evaluations but rarely results were consistent with preclinical evidence. For example, considering cyclindependent kinases 4/6 (CDK 4/6) inhibitors, preclinical data and phase 1 studies suggested that CCND1 amplification or p16 loss was predictive of response, but the phase 2 PALOMA 1 study, which was designed to specifically address this issue, showed equal benefit of palbociclib, regardless of their status in neoplastic tissue (7). On the other hand, the BELLE-2 trial showed that PIK3CA mutations in ctDNA predict the activity of buparlisib therefore suggesting that the status of this biomarker could be assessed before treatment (8). In this respect, liquid biopsy is a pivotal technology.

In conclusion, randomized phase III trials have shown that CDK4/6 inhibitors are effective in HR positive BC but newer studies including predefined biomarker-based design are needed to validate the efficacy of CDK4/6 inhibitors in molecularly defined patients' subsets. We hope that the ongoing translational efforts, building on previous failure and with the support of innovative trial design and technologies, will result in a better understanding of the genetic alterations or pathways activations to improve treatment personalization. Defining populations with a specific alteration in a given signaling pathway could improve the likelihood of benefit from a targeted agent, limit unnecessary toxicities, and allow the sustainability of these newer treatments.

\section{Acknowledgements}

None.

\section{Footnote}

Conflicts of Interest: The authors have no conflicts of interest to declare.

\section{References}

1. O'Leary B, Finn RS, Turner NC. Treating cancer with selective CDK4/6 inhibitors. Nat Rev Clin Oncol

2016;13:417-30. 
2. Hortobagyi GN, Stemmer SM, Burris HA, et al. Ribociclib as First-Line Therapy for HR-Positive, Advanced Breast Cancer. N Engl J Med 2016;375:1738-48.

3. Finn RS, Martin M, Rugo HS, et al. Palbociclib and Letrozole in Advanced Breast Cancer. N Engl J Med 2016;375:1925-36.

4. Geuna E, Milani A, Martinello R, et al. Buparlisib, an oral pan-PI3K inhibitor for the treatment of breast cancer. Expert Opin Investig Drugs 2015;24:421-31.

5. Baselga J, Campone M, Piccart M, et al. Everolimus in postmenopausal hormone-receptor-positive advanced breast cancer. N Engl J Med 2012;366:520-9.

6. Connolly R, Li H, Jankowitz RC, et al. Combination

Cite this article as: Genta S, Mittica G, Giannone G, Ghisoni E, Valabrega G. Are cyclin-dependent kinases 4/6 inhibitors ready for prime time in estrogen-receptor positive metastatic breast cancer? Transl Cancer Res 2017;6(Suppl 1):S197-S200. doi: 10.21037/tcr.2017.02.20
Epigenetic Therapy in Advanced Breast Cancer with 5-Azacitidine and Entinostat: A Phase II National Cancer Institute/Stand Up to Cancer Study. Clin Cancer Res 2016. [Epub ahead of print].

7. Finn RS, Crown JP, Lang I, et al. The cyclin-dependent kinase 4/6 inhibitor palbociclib in combination with letrozole versus letrozole alone as first-line treatment of oestrogen receptor-positive, HER2-negative, advanced breast cancer (PALOMA-1/TRIO-18): a randomised phase 2 study. Lancet Oncol 2015;16:25-35.

8. PI3K Inhibitor Improves PFS in BELLE-2 Trial. Cancer Discov 2016;6:115-6. 